\title{
Quaderni
}

QUADERNI Communication, technologies, pouvoir

80 | Hiver 2012-2013

L'intégration européenne

\section{Analyser les résistances nationales à la mise en œuvre des normes européennes : une étude des instruments d'action publique}

Sabine Saurugger et Fabien Terpan

\section{OpenEdition}

Journals

Édition électronique

URL : http://journals.openedition.org/quaderni/666

DOI : $10.4000 /$ quaderni.666

ISSN : 2105-2956

Éditeur

Les éditions de la Maison des sciences de l'Homme

Édition imprimée

Date de publication : 5 janvier 2013

Pagination : 5-24

ISBN : 978-2-7351-1525-9

Référence électronique

Sabine Saurugger et Fabien Terpan, « Analyser les résistances nationales à la mise en œuvre des normes européennes : une étude des instruments d'action publique », Quaderni [En ligne], 80 | Hiver 2012-2013, mis en ligne le 05 janvier 2015, consulté le 17 mars 2020. URL : http://

journals.openedition.org/quaderni/666; DOI : https://doi.org/10.4000/quaderni.666 


\section{$D$ ossier}

\section{Analyser les résistances} nationales à la mise en œuvre des normes européennes : une étude des instruments

\section{d'action publique}

Sabine Saurugger

Professeur de Science politique Sciences Po Grenoble, Institut universitaire de France (IUF)

Fabien Terpan

Maître de conférences en Droit public Sciences Po Grenoble, CESICE
Une abondante littérature a décrit et analysé le rôle du droit en tant que facteur d'intégration ${ }^{1}$. La construction d'une Communauté de droit a accompagné la réalisation du marché commun dès les années 1960, sur la base de principes développés par la Cour de justice des Communautés européennes, en particulier le principe de primauté du droit communautaire sur les droits nationaux, et celui de l'effet direct des traités, des règlements et des directives. À première vue, la mécanique de l'intégration juridique semble favoriser la réalisation d'une Union « sans cesse plus étroite », objectif fixé par le traité de Rome, en faisant reculer une à une les oppositions nationales selon une logique quasi-automatique. Les nombreux travaux sur l'européanisation ${ }^{2}$ montrent que cette logique est bien présente, qu'elle affecte de nombreuses politiques publiques et quasiment toutes les branches du droit. De là à conclure à un processus inexorable, insusceptible de blocages ou de retours en arrière, il y a un pas qu'on ne saurait franchir.

Depuis le début des années 1990, les différentes formes d'opposition à la mise en œuvre des normes européennes sont de plus en plus visibles. Les plaintes adressées contre les États membres sont nombreuses, certaines aboutissant à des arrêts de manquement prononcés par la Cour de justice. Une décision récente de la Cour constitutionnelle allemande insiste sur les limites du processus d'intégration, notamment en termes de démocratie, et rappelle que l'Union européenne n'a pas fait disparaître la souveraineté étatique ${ }^{3}$. D'un point de vue plus politique, d'autres résistances émergent également. Par exemple, la procédure de déficit excessif sanctionnant la non-application des critères du Pacte de stabi- 
lité et de croissance (une dette ne dépassant pas $60 \%$ du PIB et un déficit public en deçà de $3 \%$ du PIB) a été bloquée dès 2003 par la France et l'Allemagne qui en faisaient l'objet. De nombreux observateurs voient dans ces évolutions le signe que les États, leur administration et leurs citoyens résistent de plus en plus à l'intégration européenne. La quantification des résistances des acteurs nationaux, aussi bien publics que privés, est toutefois difficile à effectuer, et elle ne constitue pas l'objet de cet article. Il s'agira ici d'identifier les formes de résistances administratives à la mise en œuvre des normes européennes, de mettre à jour leur diversité sur la base d'une mise en perspective historique.

Si la littérature actuelle porte une grande attention aux différentes formes d'euroscepticisme définies comme les résistances des citoyens aux conséquences perçues de l'intégration européenne, les résistances des États et des administrations nationales font l'objet d'un intérêt moins systématique. Loin d'être récentes ou limitées à la période ouverte par le traité de Maastricht, elles sont apparues dès les premières années de la construction européenne et les traités de Paris et de Rome créant les trois Communautés européennes. La notion de résistance doit être comprise comme une notion générique qui suppose non seulement une opposition entre différentes volontés (donc une résistance active), mais aussi une absence d'action due à un manque de ressources matérielles ou de savoir (résistance passive). Son utilisation nous permet d'englober des comportements d'opposition tels que les contournements par l'action ou la nonaction, les adaptations ou les répertoires d'action protestataires $^{4}$. Le polymorphisme de la notion nous amène à dresser un inventaire des formes de résistances, ce qui constitue un enjeu tant méthodologique que théorique.

L'existence de résistances de la part des États a été mise à jour sous l'angle de la non-conformité avec le hard law (réglements, directives, décisions) : problèmes de transposition des directives, réticences du juge national à suivre les positions adoptées par la Cour de justice des Communautés européennes (aujourd'hui de l'Union européenne), manquements des États poursuivis par la Commission et sanctionnés par la Cour. Des résistances ont été décelées à tous les échelons. Celui du pouvoir politique et administratif, tout d'abord, au niveau central (gouvernement et Parlement), mais aussi au niveau local de l'État et des collectivités territoriales. Celui du pouvoir juridictionnel, ensuite, puisque le juge national est censé faire respecter la primauté et l'effet direct du droit de l'Union européenne. Toutefois, la question de la non-conformité au hard law n'est qu'une des possibilités d'analyser les résistances des États. Dans le contexte européen, les normes ne renvoient pas seulement à des actes formels et contrôlés par la Cour tels que les règlements, directives ou décisions de l'article 288 TFUE, mais aussi à des règles dites de soft law dont le non suivi n'entraine pas systématiquement une sanction juridique. Dans le cas des politiques de l'emploi, par exemple, ces règles prennent la forme d'indicateurs, d'objectifs à atteindre. Leur mise en œuvre est supervisée par le Conseil, lequel s'appuie sur un rapport émis par un Comité composé de représentants des États membres et de la Commission. Aucune sanction n'est prévue dans l'hypothèse où les objectifs ne seraient pas atteints. Ces normes de soft law, programmes et 
autres codes de conduite sont, contrairement au hard law, difficiles à observer empiriquement, mais non moins présentes dans le système de l'Union.

Dans cet article, nous démontrerons que l'opposition administrative aux normes produites par l'Union européenne n'est pas récente, mais accompagne structurellement la construction européenne depuis ses débuts. Toutefois, ces oppositions et résistances se sont partiellement transformées, en particulier à partir des années 1990, dans un contexte de remise en cause de la méthode communautaire (fondée sur l'équilibre l'institutionnel, l'importance des institutions supranationales, y compris la Cour de justice, et la primauté du droit de l'UE sur les droits nationaux), contexte dans lequel le hard law de l'Union européenne est de plus en plus complété par du soft law $w^{5}$ L'objectif de l'article est de proposer un agenda de recherche ayant pour élément central la constitution d'une typologie des instruments de résistances aux normes européennes - soft ou hard law -, typologie basée sur une mise en perspective historique des résistances nationales et de leurs évolutions.

Un instrument de résistance, selon la définition désormais classique de Lindner et Peters, élargie par Lascoumes et Le Galès, est « un dispositif à la fois technique et social qui organise des rapports sociaux spécifiques entre la puissance publique et ses destinataires en fonction des représentations et des significations dont il est porteur $»^{6}$. Cette définition spécifique de l'instrument est privilégiée car c'est la seule qui nous permette d'englober à la fois les résistances actives, décidées consciemment par les acteurs, et les résistances passives, dues à un manque de ressources matérielles ou cognitives, ou à une simple inertie. Les instruments de résistance sont donc aussi bien des stratégies que des outils dont les acteurs font usage. La typologie élaborée sur cette base fournira un cadre susceptible de guider les recherches futures portant sur les résistances administratives et dans lequel pourront s'intégrer les études de cas de ce numéro spécial portant sur les résistances dans différents domaines d'activités de l'Union.

Les États, depuis leur origine, font preuve d'une réelle inventivité dans la recherche d'instruments leur permettant de s'opposer à la mise en œuvre de normes européennes juridiquement contraignantes et sanctionnées par des juridictions. Il s'agit là de formes «traditionnelles » de résistances aux normes de hard law élaborées dans le cadre de la méthode communautaire (1). Dans le contexte de la fin du consensus permissif ${ }^{7}$, de nouveaux modes de gouvernance sont apparus, qui reposent essentiellement sur des normes de soft law. Cette évolution n'a pas tari la source des résistances, loin de là. Au contraire, les formes de résistances se diversifient au cours du temps et des évolutions normatives de l'Union (2). En tenant compte des apports et des limites des travaux portant sur les formes traditionnelles ou plus nouvelles de résistances, la section finale de cet article propose des pistes pour renforcer la recherche sur les résistances étatiques aux normes européennes (3).

\section{Conformité et non conformité aux normes de Hard Law}

Savoir comment les États et ses acteurs se mettent en conformité avec les normes internationales 
qu'ils ont adoptées, ou contribuées à adopter, est une problématique centrale qui permet de comprendre le fonctionnement de l'État mais aussi celui du système international. Décrire l'application des normes suppose d'analyser à la fois la mise en conformité -processus qui mène à une situation où le comportement de l'acteur et/ou son identité correspondent à une règle spécifique ${ }^{8}$ - et l'autre côté de la médaille, à savoir les oppositions que suscite l'exigence de conformité. Dans le cadre de la construction européenne, l'influence des normes sur les systèmes politiques et juridiques nationaux a été mise à jour dès la création des Communautés dans les années 1950, et les oppositions à ces normes sont apparues aussitôt, portées par des acteurs nationaux (1.1). Il a fallu attendre les années 1980 pour ces résistances soient étudiées de manière systématique (1.2).

\subsection{Le développement de résistances accom- pagne la mise en place d'une logique d'inté- gration juridique}

La logique de l'intégration par le droit résulte des traités de Paris et de Rome, mais aussi de l'interprétation qu'en a donnée la Cour de justice, en particulier dans ses arrêts fondateurs de 1963-649. Dès lors que les Communautés donnent naissance à un ordre juridique spécifique, distinct de l'ordre international, et composé de normes directement applicable dans les États membres et supérieures aux normes de droit interne, les États se trouvent soumis à une pression intégrative qui prend la forme d'une exigence de conformité ${ }^{10}$. Il leur appartient de supprimer les dispositions contraires au droit communautaire et de ne pas en adopter de nouvelles, d'appliquer les règles européennes pourvues d'effet direct, et de supprimer les pratiques contraires à ces règles.

Cette logique d'intégration a donné naissance à un phénomène d'européanisation du droit, qui touche les différentes branches du droit interne, mais elle a aussi produit des résistances, des refus, des oppositions à l'exigence de conformité. Les États membres, depuis l'origine, ont fait preuve d'une réelle inventivité dans la recherche d'instruments leur permettant de s'opposer à la production de normes « législatives » européennes.

Certes, les États ont l'obligation de suivre les prescriptions contenues dans les règles européennes. Mais le contexte politique interne peut amener les acteurs étatiques, individuels ou collectifs, à rejeter ces prescriptions, alors même que les institutions européennes ont été autorisées, par un transfert de compétences, à adopter des règles obligatoires. Certes, les États sont tenus de respecter un principe de coopération loyale entre eux et avec les institutions, mais ils disposent aussi d'une marge de manœuvre puisque l'application des normes obéit à un principe d'autonomie institutionnelle qui laisse les États libres de choisir les organes et procédures qui leur permettront de se mettre en conformité.

Ainsi, les résistances nationales à la mise en œuvre des normes européennes peuvent concerner tout type d'autorité publique, qu'il s'agisse du pouvoir exécutif ou législatif, du pouvoir central ou local, du pouvoir politique ou juridictionnel. Elles prendront la forme d'une abstention d'agir (directive non transposée, violation d'un règlement) ou d'une action contraire au droit de l'UE. Elles se traduiront par un acte juridique ou par une simple pratique administrative. Lorsqu'il 
s'agit d'actes juridiques, elles peuvent concerner le niveau constitutionnel (réticences des cours constitutionnelles à admettre la primauté du droit de l'UE sur les constitutions), le niveau législatif (lois contraires au droit de l'UE; refus du Conseil d'État d'admettre la primauté du droit de l'UE sur les lois postérieures jusqu'à l'arrêt Nicolo de $1989^{11}$ ) ou le niveau réglementaire.

Des années 1950 jusqu'au début des années 1980 , ce sont les domaines du marché commun et de la concurrence qui ont fourni davantage d'exemples de résistances. Le maintien d'entraves à la libre circulation des marchandises a entraîné une importante jurisprudence de la Cour sur les taxes d'effet équivalent à des droits de douane et les mesures d'effet équivalent à des restrictions quantitatives. Si les barrières tarifaires et non tarifaires ont été supprimées au $1^{\text {er }}$ juillet 1968, les gouvernements des États membres ont rapidement mis en place des règlementations, des taxes ou de simples pratiques ayant un effet équivalent à des droits de douanes ou à des restrictions quantitatives. Ces formes de résistances ont été régulièrement condamnées par la Cour sur la base de recours en manquement formés par la Commission. De la même façon, les États ont opposé des résistances à la libre circulation des personnes, en cherchant à protéger les emplois dans la fonction publique ou en s'appuyant, parfois de manière très extensive, sur les exceptions au principe de liberté figurant dans les traités et liées à la notion d'ordre public. Dans le domaine de la concurrence, l'argument de l'intérêt général ou du service public a été utilisé pour fournir des aides publiques non conformes au principe de la concurrence libre et non faussée.
Néanmoins, le marché et la concurrence ne sont pas restés les seuls domaines de résistances nationales. L'élargissement des compétences de l'Union européenne, consacré par l'Acte unique, puis par les traités de Maastricht, Amsterdam, Nice et Lisbonne, a donné naissance a des résistances nouvelles. Les rapports de la Commission sur l'application du droit de l'UE permettent de constater que les résistances aux normes de hard law concernent la plupart des politiques de l'Union. A cet égard, la répartition entre les différents domaines d'activités des recours en manquement formés devant la Cour de justice contre les États membres est particulièrement révélatrice de cette diversification. Certes, toutes les résistances ne font pas l'objet d'un recours devant la Cour de justice : il est donc possible que les statistiques relatives aux procédures de manquement ne reflètent pas exactement les pratiques administratives (cf. Tableau 1). Par exemple, la forte proportion d'affaires relatives aux questions environnementales pourrait s'expliquer par l'activisme des ONG de ce secteur, promptes à utiliser l'arme du droit, ce qui donnerait l'impression que les résistances administratives sont plus importantes qu'elles ne le sont réellement. Néanmoins, quel que soit l'écart existant entre les résistances et les saisines de la Cour, les statistiques du recours en manquement fournissent une information utile sur la diversité des domaines de friction entre normes européennes et pratiques nationales. 
Tableau 1 : Recours en manquement 2010

\begin{tabular}{|l|l|}
\hline Protection de l'environnement & $19,77 \%$ \\
\hline Marché intérieur et services & $17,73 \%$ \\
\hline Justice & $14,4 \%$ \\
\hline Fiscalité et union douanière & $11,1 \%$ \\
\hline Emploi et affaires sociales & $8,91 \%$ \\
\hline Entreprises et industrie & $4,72 \%$ \\
\hline $\begin{array}{l}\text { Santé et protection des } \\
\text { consommateurs }\end{array}$ & $3,5 \%$ \\
\hline Concurrence & $3 \%$ \\
\hline Mobilité et transport & $2,64 \%$ \\
\hline Affaires intérieures & $2,05 \%$ \\
\hline Énergie et transports & $1,45 \%$ \\
\hline
\end{tabular}

Source : Report from the European Commission, 28th Annual Report on Monitoring the Application of EU Law, 2010, COM(2011)588 final, Statistical Annex, Annexes I-III

\subsection{Une approche plus systématique dans l'analyse des mécanismes de conformité / non conformité}

Dans un premier temps, les résistances nationales ont été mises à jour par l'étude de la jurisprudence de la Cour de justice, laquelle contrôle la conformité des droits internes par rapport au droit de l'Union européenne. Les travaux, essentiellement juridiques, sur l'application des règles européennes, ont permis de constater la grande diversité des formes de résistances et les ont catégorisées à partir des règles européennes elles-mêmes, et non à partir des instruments de résistances utilisés. C'est ainsi qu'on a pu identifier l'existence de charges pécuniaires ne respectant pas l'interdiction des droits de douanes ou encore l'existence de formalités administratives contraires à la liberté de circulation ou à la liberté d'établissement au sein de l'Union européenne ${ }^{12}$. Jusqu'aux années 1980, on observe peu d'efforts dans l'analyse systématique des types de résistance ${ }^{13}$. Aucune typologie transversale -c'est-àdire transcendant les domaines de compétences et les politiques publiques européennes- n'a été proposée. Peu d'explications ont été fournies sur la résistance des États à l'exigence de conformité. Sinon que le droit communautaire n'est pas plus mal mis en œuvre que n'importe quelle loi nationale, ce qui paraît logique dès lors que l'administration et encore plus les citoyens ne sont pas nécessairement informés de la provenance d'une règle qu'ils appliquent ${ }^{14}$.

À partir des années 1980, des analyses - essentiellement de science politique- ont tenté d'étudier la question de la conformité de manière plus systématique. Deux catégories de travaux doivent être évoquées.

La première est composée d'études portant sur la transposition des directives à l'échelle de l'État. Il s'agissait de comprendre dans quelle mesure les États membres transposent en droit interne les règles contenues dans les directives européennes ${ }^{15}$. Leur inclusion dans les législations et réglementations nationales a été mesurée par leur apparition dans les journaux officiels des États membres. Plus le délai entre la publication au Journal Officiel de l'UE et celle réalisée au niveau national était limité, plus la conformité avec la législation européenne était considérée comme grande. Cette analyse s'appuyait implicitement sur l'hypothèse que les transpositions de directives mènent à une convergence accrue entre les différents États membres. ${ }^{16}$ 
À partir du milieu des années 1990, on voit émerger une seconde catégorie d'études, s'attachant à décrire et analyser les différents degrés de mise en conformité au plan national, incluant aussi bien la transposition des directives, que le respect des règlements, des traités et des décisions ${ }^{17}$. Les différences entre les États membres deviennent un objet d'études, ou formulé autrement, une variable dépendante explicable par des configurations institutionnelles facilitant ou au contraire empêchant la mise en œuvre des normes européennes ${ }^{18}$. Deux formes de résistances sont alors identifiées : la résistance passive (ou inertie) et la résistance active (cf. tableau 1$)$.

Alors que l'inertie renvoie à une absence de changement (ou une abstention d'action, comme le nomment les travaux juridiques évoqués plus haut), une situation où les normes européennes n'entraînent aucune modification de la politique d'un pays, d'autres formes de résistance se traduisent, dès le début de la mise en œuvre, par une action nationale contraire à la norme européenne ${ }^{19}$. Dans ce cas, l'opposition aux décisions européennes crée, au niveau national, des coalitions qui imposent des réformes allant à l'encontre de celles préconisées au niveau européen. En majorité, les travaux sur la nonconformité avec le droit européen se présentent sous forme d'études de cas s'appuyant sur des méthodes qualitatives ${ }^{20}$, sur des méthodes à la fois qualitatives et quantitatives ${ }^{21}$, ou sur des méthodes purement quantitatives ${ }^{22}$.

Les études distinguent généralement les variables qui affectent, de manière positive, la mise en conformité du droit national avec le droit de l'UE, et celles qui empêchent, de manière négative,
Tableau 2 : Résistances actives et passives ${ }^{23}$

\begin{tabular}{|l|l|}
\hline Résistances actives & Résistances passives \\
\hline $\begin{array}{l}\text { Opposition contre } \\
\text { des dispositions pré- } \\
\text { cises }\end{array}$ & $\begin{array}{l}\text { Interprétations diver- } \\
\text { gentes }\end{array}$ \\
\hline $\begin{array}{l}\text { Opposition contre le } \\
\text { mode décisionnel au } \\
\text { niveau européen }\end{array}$ & $\begin{array}{l}\text { Difficultés adminis- } \\
\text { tratives (manque de } \\
\text { ressources, de forma- } \\
\text { tion) }\end{array}$ \\
\hline $\begin{array}{l}\text { Opposition contre } \\
\text { le mode de trans- } \\
\text { position au niveau } \\
\text { national }\end{array}$ & Instabilité politique \\
\hline $\begin{array}{l}\text { Conflits intra - ou } \\
\text { inter - ministérielles }\end{array}$ & \\
\hline
\end{tabular}

une transposition efficace. Plusieurs variables affectent le degré de mise en conformité de manière positive, en particulier l'efficacité administrative, la capacité de contrôle de l'assemblée parlementaire et son pouvoir de coordination. Les variables qui influencent la mise en conformité de manière négative sont : la mise en œuvre plus ou moins décentralisée, le niveau de corruption, le nombre de veto players (aussi bien publics que privés), le degré de conflictualité politique au niveau national (cf. tableau 2).

Incontestablement, les travaux évoqués supra ont atteint un degré élevé de sophistication théorique et méthodologique, même si les résultats obtenus sont peu cumulatifs ${ }^{24}$. La plupart des travaux, en particulier qualitatifs, portent sur un secteur de 
politique publique en particulier. Ces recherches s'intéressent généralement à l'une ou l'autre forme de résistance-passive ou active-, mais leur méthodologie ne permet pas de comprendre dans quel contexte politique spécifique l'une ou l'autre des résistances a lieu prioritairement. Ainsi, les recherches de Falkner et al. montrent l'existence de différentes formes nationales d'oppositions aux normes européennes dans le domaine de la politique sociale, en insistant sur le critère principal du contexte institutionnel national (les États nordiques transposent les directives en temps et en heure, alors que les États du Sud, en particulier la Grèce, l'Italie et la France sont systématiquement en retard et s'opposent plus souvent $)^{25}$. Dans le secteur de la protection des consommateurs, les travaux de Versluis montrent que le facteur principal est, au contraire, la visibilité politique : plus la visibilité politique d'un problème est élevée, plus les résistances augmentent ${ }^{26}$.

Tableau 3 : Variables influençant le niveau de conformité $^{27}$

\begin{tabular}{l|l} 
Effet positif & Effet négatif \\
\hline $\begin{array}{l}\text { Efficacité admi- } \\
\text { nistrative }\end{array}$ & $\begin{array}{l}\text { Processus décisionnels } \\
\text { décentralisés } \\
\text { Contrôle parle- } \\
\text { mentaire }\end{array}$ \\
$\begin{array}{l}\text { Nouveau de corruption } \\
\text { coordination du } \\
\text { parlement }\end{array}$ & $\begin{array}{l}\text { Veto players (publics et } \\
\text { privés) } \\
\text { Degré de conflictualité } \\
\text { politique }\end{array}$
\end{tabular}

Ces travaux se sont essentiellement concentrés sur la mise en œuvre des actes juridiques contrai- gnants que sont les directives et les règlements. Comme on va le voir, les traités européens ont été modifiés de telle sorte que le processus d'intégration emprunte aujourd'hui de nouvelles voies, qui remettent en cause - sans la supprimer ${ }^{28}$ - la traditionnelle méthode communautaire. Loin d'éliminer ou de contourner les résistances nationales, ces nouvelles voies ont, à leur tour, donné naissance à des oppositions, rejets, inactions qui doivent être prises en compte si l'on veut proposer une typologie complète des instruments de résistance.

\section{Les nouvelles formes de résistances liées au développement du soft law dans l'Union européenne}

Le début des années 1990 apparaît comme une période charnière pour deux raisons principales. Premièrement, c'est une période qui correspond à la fin du consensus permissif ${ }^{29}$ en vertu duquel les citoyens faisaient confiance aux gouvernants pour gérer les affaires européennes et soutenaient le processus d'intégration sans s'y intéresser véritablement. Des formes de résistances se manifestent chez les citoyens qui interagissent avec les formes de résistances existant au niveau des États et de leur administration. Deuxièmement, c'est à ce moment qu'apparaissent les « nouvelles formes de gouvernance », essentiellement fondées sur du soft law, c'est-à-dire sur des normes dépassant l'intégration juridique au sens classique (primauté et effet direct des directives et règlements) et pour lesquelles les institutions européennes n'ont que de faibles pouvoirs coercitifs (excluant le contrôle juridictionnel par la Cour de justice). Ces nouveaux modes de gouvernance ont été mis en place et se sont développés dans l'espoir 
qu'ils permettraient de contourner les résistances étatiques (2.1). Celles-ci n'ont pas disparu, cependant, comme les études de cas réunies dans ce numéro le démontrent, et elles doivent donc être étudiées autant que les résistances aux normes de hard law (2.2).

\subsection{Contourner les résistances étatiques par le développement des normes de soft law}

Deux types de règles composent la catégorie $s o f t$ $l a w^{30}$. D'une part, des règles contraignantes qui échappent au hard law parce qu'elles ne sont pas placées sous le contrôle de la Cour (cas des décisions dans le domaine de la Politique étrangère et de sécurité commune ou du Pacte de stabilité et de croissance). D'autre part, des règles non contraignantes sont considérées comme relevant du soft law parce qu'elles fixent des objectifs et prévoient des mécanismes permettant d'encadrer leur réalisation. Ce second type de normes est en fort développement depuis les années 1990, en particulier dans les domaines de la coordination économique (depuis le traité de Maastricht), de la politique de l'emploi (depuis le traité d'Amsterdam) ou des retraites et de l'inclusion sociale (depuis la stratégie de Lisbonne). Ces politiques s'appuient sur des modalités telles que le benchmarking (l'étalonnage), les best practices (meilleures pratiques), le peer review (évaluation par les pairs) ou encore le mainstreaming (modes d'action horizontale), qui s'ajoutent aux modalités originelles que constituent les règles de hard law (traités, principes généraux d'origine jurisprudentielle, règlements, directives, décisions). Les nouvelles modalités ont ceci de particulier qu'elles permettent aux États de mettre en place des approches volontaires de la régulation ${ }^{31}$.
Elles prennent place dans le cadre de processus non cœrcitifs qui s'appuient sur la volonté des participants de se mettre d'accord par la délibération collective, sur des normes procédurales, des modes de régulation et des objectifs politiques communs, tout cela en préservant la diversité des solutions, à l'échelle nationale voire locale ${ }^{32}$. L'objectif de ces formes de gouvernance n'est pas de créer des normes juridiquement contraignantes auxquelles tous les États membres devraient se conformer, mais de permettre aux États de préserver leurs spécificités nationales tout en les rendant compatibles avec les priorités politiques et économiques de l'Union européenne. Ainsi l'outil juridique utilisé n'est pas le règlement et la directive mais des instruments non contraignants relevant du volontariat ou du soft law. Ces instruments ne sont pas directement applicables en droit interne. Ils sont négociés entre acteurs publics et privés, à différents niveaux du processus décisionnel, le choix politique effectif des modalités d'application étant laissé aux États membres $^{33}$ selon une logique relevant du principe de subsidiarité.

Si les politiques publiques couvertes par la méthode ouverte de coordination (emploi, coordination des politiques économiques, inclusion sociale et retraites) sont au cœur du soft law européen, d'autres formes de coordination donnent naissance à des normes non contrôlées par la Cour, dans des domaines nombreux et variés : éducation, culture, recherche et développement, industrie, concurrence, environnement, énergie, etc. Les documents tels que la stratégie de Lisbonne ou la stratégie 2020 renvoient en grande partie à des normes de soft law $w^{34}$. Ces dernières se traduisent par des accords volontaires, des stan- 
dards, des labels et des mécanismes diversifiés d'incitation financière et fiscale, qui renvoient très fortement aux instruments développées dans le contexte du new public management. Ils constituent des critères que les autorités nationales acceptent de prendre en compte dans la formation de leurs propres politiques ${ }^{35}$.

Deux raisons, liées au degré attendu de résistance de la part des acteurs nationaux, expliquent l'émergence des ces normes :

1. Les nouveaux modes de gouvernance entraineraient davantage de cohérence entre les États membres de l'UE parce que le mécanisme premier mis en place par ce type de norme est l'apprentissage. Cet argument, présent dans de nombreux documents officiels de la Commission européenne, tels que le Livre Blanc sur la Gouvernance de 2001, insiste sur la diversité des formes d'apprentissage (socialisation, observation et mimétisme, délibération et conviction), lesquelles réorientent les paradigmes initiaux et les positions des acteurs. La résistance dans ce contexte serait très faible.

2. Les gouvernements, tout comme les institutions européennes, produiraient une « ombre de hiérarchie » (shadow of hierarchy) en menaçant d'introduire des réglementations contraignantes si les acteurs ne se mettent pas en conformité avec les instruments volontaires ou relevant $\mathrm{du}$ soft law ${ }^{36}$.

\subsection{Mesurer les résistances aux normes de Soft law}

Il est cependant possible, comme plusieurs exemples dans ce numéro spécial le montrent, que des attitudes de résistance apparaissent même dans des contextes de soft law. Étant donné l'absence de contrôle juridictionnel sur les règles de soft law, elles apparaissent d'ailleurs en permanence puisqu'aucune sanction ne s'applique en cas de non-respect de la norme. On observe donc le développement de formes particulièrement innovantes de résistance parce que les acteurs sont libres d'utiliser les instruments à leur guise. Toutefois, les résistances prennent moins la forme d'une opposition directe que celle d'une orientation plutôt indirecte induisant un changement incrémental ${ }^{37}$. Le plus souvent, des stratégies de résistance se déploient de manière masquée, au stade de la mise en œuvre, alors même qu'un accord de principe a été trouvé sur les grands objectifs.

Ainsi, les actes de soft law, créés pour convaincre les États qu'ils peuvent faire converger leurs politiques sans avoir besoin de réaliser d'importantes limitations de souveraineté, font l'objet de résistances aussi importantes que les actes de hard law. Les États membres, à l'échelon politique ou administratif, ne reprennent pas facilement à leur compte les incitations non contraignantes relevant de la méthode ouverte de coordination ou les règles contraignantes qui ne sont pas assorties de contrôle juridictionnel.

Mesurer la résistance aux normes de hard law dont nous avons parlé dans la section 1 entraine des problèmes méthodologiques et théoriques nombreux : quand peut-on dire qu'une transposition de directive a été pleinement réalisée ? Une directive peut formellement avoir été transposée en droit interne sans pour autant que la mise en 
œuvre soit considérée comme efficace ou satisfaisante. Différentes causes expliquent ce genre de situation : Versluis pointe du doigt l'absence d'attention médiatique et donc de pression sur l'administration pour mettre en œuvre les dispositifs ; Falkner et al attribuent la responsabilité de ces difficultés à un manque de ressources administratives.

Si analyser les résistances aux normes contraignantes pose déjà des problèmes sérieux, l'administration de la preuve est encore plus délicate pour le soft law et les nouveaux modes de gouvernance. Un pas vers la résolution de ce problème est possible en portant notre attention sur la manière dont les acteurs font usage des différentes formes de résistance, plutôt qu'en se focalisant sur le nombre de dispositifs non transposés ou non mis en œuvre. Cette approche nous invite à comprendre les usages des instruments de résistances, et donc le comportement des acteurs dans les phases de mise en œuvre du soft law. Ce sont donc les attitudes de résistance des acteurs nationaux à travers les instruments qu'ils mettent en œuvre qu'il convient d'analyser.

\section{Mieux analyser les résistances à travers l'étude des instruments}

Il ressort de ce qui précède que l'analyse des résistances peut être renforcée de trois manières différentes.

Premièrement, il s'agit d'étudier de façon plus approfondie les résistances aux nouvelles formes de gouvernance, qui font l'objet de travaux trop peu nombreux. Partant de la définition du soft law développée plus haut, il importe ainsi de réper- torier les différentes formes de soft law existant au sein de l'Union européenne. A partir de là, il convient d'analyser en profondeur les principaux domaines d'application du soft law à l'échelle de l'UE, en s'intéressant à la méthode ouverte de coordination, mais aussi aux documents stratégiques (type stratégie de Lisbonne et stratégie 2020) ou à certains aspects de la politique étrangère et de sécurité commune.

Cette analyse permettra de vérifier de manière systématique quels types de résistances s'appliquent à quels types de politiques publiques européennes. Une base de données des dispositifs relevant du soft law européen ouvrira la possibilité d'engager une comparaison des types de mises en œuvre de ces normes dans les différents États membres de l'Union européenne. Une typologie distinguant entre les États membres selon qu'ils sont bons ou mauvais élèves, en queue ou en tête de peloton de la mise en ouvre des normes européennes (laggards and champions), pourra ainsi être interrogée sur des bases empiriques solides. Ceci nous permettra de comprendre, précisément, s'il existe les mêmes phénomènes de résistance à la mise en œuvre du soft law et du hard law, ou si des différences persistent entre les États membres.

Deuxièmement, il apparaît nécessaire d'élaborer une typologie des instruments de résistances, aussi bien en termes d'opposition au hard law qu'au soft law de l'Union européenne.

Comme nous l'avons souligné plus haut, l'approche sociologique des instruments nous permet d'affiner la typologie existant dans la littérature qui distingue schématiquement entre 
résistances actives et passives. N'envisageant pas les instruments de résistance comme des réponses fonctionnelles à des normes européennes considérées comme non-souhaitables par les acteurs nationaux, l'approche des instruments de politique publique permet d'introduire la variable du contexte, aussi bien institutionnel que politique ou social, dans l'analyse des résistances aux normes européennes. Ainsi, les oppositions aux normes et le choix des instruments de résistances sont le résultat des jeux d'acteurs, des rapports de force à un moment donné, lesquels sont fortement influencés par le contexte institutionnel, par la situation politique nationale, et par le type de normes auxquelles on s'oppose. En termes de sociologie pragmatique, l'opposition à la mise en œuvre d'une norme européenne n'est pas vue comme la matérialisation d'une «idée initiale, mais comme une dynamique souvent chaotique de mise en convergence d'informations, d'adaptation à des contraintes et d'arbitrage entre des voies de développement divergents $»^{38}$.

Il est possible de distinguer ici entre quatre types d'instruments de résistance : les instruments juridiques, économiques/fiscaux, informatifs et communicationnels, instruments dont l'usage sera illustré par plusieurs études de cas incluses dans ce numéro spécial (cf. tableau 4). Ainsi, les contributions de Charlotte Halpern ainsi que de Mathieu Ansaloni et Virginie Tournay montrent comment une combinaison entre instruments juridiques, économiques et informatifs a permis aux administrations nationales de contourner les oppositions des acteurs centraux du traitement des déchets et de l'agro-alimentaire, dont la collaboration est indispensable pour mettre en œuvre les programmes européens. Leurs études insistent sur la nécessité de décentrer l'analyse des effets de l'intégration européenne de la seule explication en termes d'européanisation. Elles illustrent plutôt les effets en termes de recomposition de l'action publique, et de trajectoires de changement dans la politique des déchets et de l'agro-alimentaire.

Les articles d'Hélène Caune et de Benjamin Lemoine, quant à eux, s'inscrivent pleinement dans la logique des résistances au soft law. Les deux textes, portant sur l'usage des instruments informatifs, montrent comment, par une réinterprétation du cadre normatif européen, des spécificités sectorielles autant que nationales peuvent perdurer. L'opposition n'est pas frontale, il s'agit de contourner mais aussi de détourner les normes pour permettre une continuité des politiques publiques initialement en place dans les deux secteurs analysés ; ou le cas échéant d'utiliser les contraintes molles de l'Union européenne pour mieux asseoir son avantage dans un rapport de force entre acteurs.

Enfin, la contribution d'Amandine Crespy se situe, elle, en amont de la mise en œuvre. La présence de cette étude dans ce numéro est indispensable car elle permet d'identifier les usages des instruments de résistances dans un conflit particulièrement saillant pour l'intégration européenne. Il semble que, contrairement à la phase de mise en œuvre des normes européennes, les résistances durant la phase de décision prennent la forme de contestations et utilisent majoritairement des instruments communicationnels et informationnels. Ainsi, Crespy montre comment la politisation de la libéralisation des services a permis une modification cruciale des rapports de 
Tableau 4 : Instruments de résistance

\begin{tabular}{|c|c|c|c|}
\hline \multicolumn{2}{|c|}{ Type d'instrument } & \multirow{2}{*}{$\begin{array}{l}\text { Acteurs principaux } \\
\text { Gouvernements/Parlements } \\
\text { Administrations (État/ collectivités locales) } \\
\text { Acteurs privés (entreprises, associations } \\
\text { citoyens) dans la mesure où il mobilisent } \\
\text { des acteurs publics (administrations et } \\
\text { tribunaux) }\end{array}$} & \multirow{2}{*}{$\begin{array}{l}\text { Illustrations empiriques } \\
\text { (issues du numéro } \\
\text { spécial) } \\
\text { Ansaloni et Tournay, } \\
\text { Halpern }\end{array}$} \\
\hline $\begin{array}{l}\text { Instruments } \\
\text { juridiques }\end{array}$ & $\begin{array}{l}\text { - Constitutionnels } \\
\text { - Législatifs et réglemen- } \\
\text { taires } \\
\text { - Décisions administratives } \\
\text { - Pratiques administratives } \\
\text { - Recours devant le juge }\end{array}$ & & \\
\hline $\begin{array}{l}\text { Instruments } \\
\text { économiques } \\
\text { et fiscaux }\end{array}$ & $\begin{array}{l}\text { - taxes } \\
\text { - attributions de finance- } \\
\text { ment } \\
\text { - relations commerciales }\end{array}$ & $\begin{array}{l}\text { Gouvernements/Parlements } \\
\text { Administrations (État/ collectivités locales) } \\
\text { Entreprises }\end{array}$ & $\begin{array}{l}\text { Ansaloni et Tournay, } \\
\text { Halpern }\end{array}$ \\
\hline $\begin{array}{l}\text { Instruments } \\
\text { informatifs }\end{array}$ & $\begin{array}{l}\text { - expertise } \\
\text { - statistiques }\end{array}$ & $\begin{array}{l}\text { Gouvernements/Parlements } \\
\text { Administrations (État/ collectivités locales) } \\
\text { Entreprises, associations } \\
\text { Citoyens }\end{array}$ & $\begin{array}{l}\text { Caune, } \\
\text { Créspy, Ansaloni et } \\
\text { Tournay, } \\
\text { Lemoine }\end{array}$ \\
\hline $\begin{array}{l}\text { Instruments } \\
\text { communica- } \\
\text { tionnels }\end{array}$ & $\begin{array}{l}\text { - scandalisation } \\
\text { - publicisation }\end{array}$ & $\begin{array}{l}\text { Gouvernements/Parlements } \\
\text { Administrations (État/ collectivités locales) } \\
\text { Médias, } \\
\text { Citoyens } \\
\text { associations }\end{array}$ & $\begin{array}{l}\text { (Lemoine) } \\
\text { Crespy }\end{array}$ \\
\hline
\end{tabular}

force entre tendances politiques. Son analyse met l'accent sur les modalités concrètes de la politisation, et permet ainsi d'identifier les conditions matérielles de diffusion d'un discours politique dans un système multiniveaux comme celui de l'Union européenne.

Au-delà de cette typologie, il faut aussi analyser l'utilisation des instruments, ou leur fonction, afin de révéler les transformations qui affectent les normes européennes, et l'intégration européenne de manière générale. À cet égard, si la distinction entre formes actives et passives de résistance ${ }^{39}$, telle qu'introduite par la recherche sur la nonconformité avec la loi européenne, nous semble utile, elle tend à marquer une différence nette entre résistances volontaires et non-volontaires qui nous semble difficile à prouver empiriquement. Par exemple, lorsqu'une administration justifie l'inertie par un manque de ressources, il peut y avoir, derrière l'apparence d'une résistance non-volontaire, une véritable stratégie de contournement de la norme.

Plutôt que de reprendre la distinction entre résistances passives et actives, il parait judicieux de 
croiser la typologie des instruments de résistance avec les trois formes sous lesquelles se manifestent les instruments de résistance (cf. tableau 5) : contestation, contournement et détournement (les deux dernières peuvent être également non-volontaires $)^{40}$. La contestation renvoie au refus affiché de la norme, accompagné d'une opposition frontale à laquelle Lascoumes et Le Bourhis attribuent une fonction de "voice $»^{41}$. Le contournement entraine de la part des acteurs un non-usage et l'utilisation d'une autre norme à la place. Elle peut permettre de neutraliser la norme à laquelle les acteurs s'opposent, et cette neutralisation peut être permanente ou temporaire. Enfin, le détournement utilise la norme européenne, mais en offre une interprétation profondément divergente de celle initialement envisagée par le législateur, comme l'illustrent en particulier les contributions de Charlotte Halpern et de Benjamin Lemoine.

\section{Tableau 5 : Formes de résistance}

\begin{tabular}{|c|c|}
\hline $\begin{array}{l}\text { Formes de } \\
\text { résistance }\end{array}$ & \\
\hline Contestation & $\begin{array}{l}\text { Refus frontal de la norme. } \\
\text { Opposition ouverte à l'exis- } \\
\text { tence et l'usage de la norme }\end{array}$ \\
\hline $\begin{array}{l}\text { Contourne- } \\
\text { ment }\end{array}$ & $\begin{array}{l}\text { Non-usage de la norme. Op- } \\
\text { position dissimilée et rempla- } \\
\text { cement par un autre norme. } \\
\text { Application d'une norme } \\
\text { pré-existante contraire. }\end{array}$ \\
\hline $\begin{array}{l}\text { Détourne- } \\
\text { ment }\end{array}$ & $\begin{array}{l}\text { Appropriation de la norme } \\
\text { pour d'autres objectifs qu'ini- } \\
\text { tialement prévus. }\end{array}$ \\
\hline
\end{tabular}

\section{Tableau 6}

\begin{tabular}{|c|c|c|c|c|}
\hline \multicolumn{2}{|l|}{ Type d'instrument } & contestation & contournement & détournement \\
\hline $\begin{array}{l}\text { Instruments juri- } \\
\text { diques }\end{array}$ & $\begin{array}{l}\text { - Constitutionnels } \\
\text { - Législatifs et régle- } \\
\text { mentaires } \\
\text { - Pratiques adminis- } \\
\text { tratives } \\
\text { - Décisions adminis- } \\
\text { tratives } \\
\text { - Contrats de droit } \\
\text { privé }\end{array}$ & $\begin{array}{l}+++ \\
+++ \\
+++ \\
+++ \\
---\end{array}$ & $\begin{array}{l}+++ \\
+++ \\
+++ \\
+++ \\
+++\end{array}$ & $\begin{array}{l}--- \\
+/- \\
+++ \\
+++ \\
+/-\end{array}$ \\
\hline $\begin{array}{l}\text { Instruments } \\
\text { économiques et } \\
\text { fiscaux }\end{array}$ & $\begin{array}{l}\text { - taxes } \\
\text { - attributions de finan- } \\
\text { cement }\end{array}$ & $\begin{array}{l}+++ \\
+++\end{array}$ & $\begin{array}{l}+++ \\
---\end{array}$ & $\begin{array}{l}+++ \\
+++\end{array}$ \\
\hline $\begin{array}{l}\text { Instruments infor- } \\
\text { matifs }\end{array}$ & $\begin{array}{l}\text { - expertise } \\
\text { - statistiques }\end{array}$ & $\begin{array}{l}+++ \\
+++\end{array}$ & $\begin{array}{l}+++ \\
+++\end{array}$ & $\begin{array}{l}+++ \\
+++\end{array}$ \\
\hline $\begin{array}{l}\text { Instruments com- } \\
\text { munica-tionnels }\end{array}$ & $\begin{array}{l}\text { - scandalisation } \\
\text { - publicisation }\end{array}$ & $\begin{array}{l}+++ \\
+++\end{array}$ & $\begin{array}{l}-- \\
---\end{array}$ & $\begin{array}{l}--- \\
+++\end{array}$ \\
\hline
\end{tabular}


En croisant les deux tableaux, nous pouvons évaluer le degré de probabilité des fonctions de résistance pour chaque instrument utilisé (cf. tableau 6).

La troisième façon de renforcer la recherche sur les résistances consiste à développer des hypothèses sur le comportement des acteurs et à les vérifier à travers des études de cas. Si les travaux présents dans ce numéro se sont concentrés sur la typologie des instruments mis en œuvre, des recherches futures permettront de vérifier la validité des hypothèses développées ci-dessous. En effet, les résistances n'arrivent pas de manière aléatoire. Il est possible, en s'appuyant sur les travaux portant sur la non-conformité avec le droit de l'UE, de développer trois hypothèses permettant de voir dans quels contextes les résistances aux normes européennes - aussi bien de hard law que de soft law - apparaissent. Chaque cas de figure permet d'évaluer la probabilité de l'hypothèse.

H1: Plus le nombre d'acteurs est élevé, plus la probabilité qu'il y ait des actions de résistance est grande. L'augmentation du nombre d'acteurs rend beaucoup plus complexe la mise en œuvre des normes et ouvre ainsi davantage de possibilités de veto. Plus il y a d'agences, de services administratifs et d'acteurs non-étatiques (groupes d'intérêt) associés au processus, plus la forme que prend ce processus est complexe. Ceci augmente les possibilités de ralentissement de la mise en œuvre, voire de blocage ou de contournement des normes par d'autres dispositifs ${ }^{42}$.

$\mathrm{H} 2$ : Plus les ressources financières et sociales ${ }^{43}$ d'un acteur sont élevées, plus leur capacité de résister est forte. L'importance des ressources permet aux acteurs de contourner les normes d'une manière particulièrement innovante. Cette hypothèse est contrefactuelle à celle émise par les études sur la non-transposition des directives - selon lesquelles le manque de ressources d'un acteur, et plus spécifiquement d'une administration, explique le faible niveau de transposition et de mise en œuvre. Nous partons au contraire de la prémisse que les attitudes de résistance résultent d'un choix stratégique et actif fait par des acteurs capables de hiérarchiser leurs objectifs. Ainsi, décider de ne pas mettre en œuvre une politique ou un instrument d'action publique peut être vu comme une attitude de résistance.

H3: Plus la distance entre les objectifs du dispositif préconisé par la norme et ceux de l'administration est grande, plus la probabilité est forte que l'acteur résiste à la mise en œuvre de ce dispositif. Ainsi, le cadre cognitif ${ }^{44}$ dans lequel évolue l'acteur est une variable déterminante du degré de résistance de l'administration. La probabilité de voir les préconisations d'une norme européenne mises en œuvre, en particulier par un acteur administratif dont le cadre cognitif est très éloigné de celles-ci, est particulièrement faible.

Ces hypothèses ne sont pas exclusives et doivent être combinées pour saisir la complexité des attitudes de résistance des acteurs. Par exemple, dans le contexte de l'Union économique et monétaire, les ministères des finances sont sans doute les acteurs possédant les plus grandes ressources. Mais on pressent qu'elles ne devraient pas opposer de résistances à la mise en place des budgets de rigueur préconisés par le pacte de stabilité et de croissance, puisque leur 'disposition' ou leurs cadres cognitifs, sont similaires à ceux du pacte. 


\section{Conclusion}

Nous l'avons vu, les oppositions ainsi que les résistances à l'intégration européenne ont une origine aussi lointaine que le projet européen luimême. Les formes que revêtent ces oppositions sont multiples, mais elles ne sont pas aléatoires.

Si nous maintenons notre hypothèse selon laquelle les résistances ont lieu indépendamment des normes qui sont en jeu - qu'elles soient juridiquement contraignantes ou non, assorties ou non d'un contrôle juridictionnel - nous constatons néanmoins que les oppositions aux normes juridiquement contraignantes peuvent davantage puiser dans l'arsenal complet des instruments de résistance (voir Ansaloni et Tournay ainsi que Halpern dans ce numéro), alors que les normes non - juridiquement contraignantes ne peuvent qu'exceptionnellement avoir recours qu'aux instruments législatifs, réglementaires et constitutionnels (voir Caune dans ce numéro).

Par ailleurs, les études de cas sur les politiques de l'emploi, la directive de libéralisation des services, ou encore les politiques environnementales montrent que l'expertise est un instrument particulièrement important pour créer de la résistance. Ce qui importe, c'est de comprendre comment un discours d'expertise peut l'emporter sur un autre. Une des explications pourrait se trouver dans le degré d'hétérogénéité du réseau d'acteurs : plus le réseau d'acteurs est hétérogène, plus la nouvelle expertise peut être captée par des acteurs dominants.

Analyser les résistances et les oppositions par les instruments sur lesquel s'appuient les acteurs nous permet d'identifier ces acteurs, de mieux saisir leur rationalité, les intérêts et cadres cognitifs divers qui entrent en jeu lors d'un processus d'opposition - qu'ils soient administratifs, juridiques ou portés par la mobilisation d'acteurs privés - et de comprendre les rapports de force entre ces acteurs. Il ne s'agit pas de tomber dans un piège " normatif » en considérant la nonconformité comme un problème à résoudre (car elle rend le système européen inefficace) mais de mieux appréhender les significations politiques dont les instruments de résistance sont porteurs. Étudier les instruments de la résistance met en cause le discours sur la dépolitisation du système politique, aussi bien national qu'européen, par l'introduction des mécanismes du New Public Management. Analyser le choix des instruments de résistances - telle expertise plutôt qu'une autre, telle forme statistique plutôt qu'une autre, telle pratique administrative plutôt qu'une autrepermet de voir à quel point le système politique reste politisé, au delà de son apparence technique ou technocratique.

Enfin, cette approche nous permet de ne pas voir dans les résistances ou oppositions aux normes européennes une attitude statique de la part des acteurs. Au contraire, une analyse du temps long dans lequel s'inscrivent les instruments de la résistance offre une perspective dynamique des résistances - les normes développées au niveau national et qui remplacent les normes européennes sont ainsi réintroduites au niveau européen. Ces dynamiques fondamentalement politiques permettent les réformes des politiques publiques. C'est cette politisation que les études sur la non-conformité - présentées plus haut - ne peuvent pas saisir. 
$\mathrm{N} \cdot \mathrm{O} \cdot \mathrm{T} \cdot \mathrm{E} \cdot \mathrm{S}$

1. Parmi les travaux de référence on peut citer M. Cappelletti, M. Seccombe \& J. Weiler (eds), Integration Through Law, Vol. 1, Book 1 and 2, Walter de Gruyter, Berlin-New York 1986, 1987.

2. Sur l'européanisation en général, voir les deux ouvrages classiques : Maria Green Cowles, James Caporaso et Thomas Risse (eds), Europeanization and Domestic Change, Ithaca, Cornell University Press, 2001; Kevin Featherstone et Claudio M. Radaelli (eds), The Politics of Europeanization, Oxford, Oxford University Press, 2003. Sur l'européanisation du droit en particulier: Francis Snyder, The Europeanization of Law, Oxford, Hart, 2000.

3. Cour constitutionnelle allemande, décision du 30 juin 2009 sur le traité de Lisbonne. Auparavant, la Cour de Karlsruhe avait pris position dans deux arrêts célèbres dits So Lange I (29 mai 1974) et So Lange II (22 octobre 1986). L'arrêt So Lange II, bien que plus souple que l'arrêt So Lange I, indique que le contrôle constitutionnel des actes européens de droit dérivé n'est pas nécessaire « aussi longtemps que » la jurisprudence de la CJCE garantit une protection des droit fondamentaux qui soit essentiellement équivalente à celle prescrite comme impérative et inaliénable par la loi fondamentale. Dès lors que ce n'est pas le cas, elle ne s'interdit pas d'exercer ce contrôle.

4. Sabine Saurugger, "Beyond non-compliance with legal norms" in Theofanis Exadaktylos and Claudio Radaelli (eds), Research design in European Studies: Establishing Causality in Europeanization, Basingstoke, Palgrave, 2012, pp. 105-124.

5. Mark Dawson, New Governance and the Transformation of European Law: Coordinating EU Social Law and Policy, Cambridge, Cambridge University Press, 2011 ; Stijn Smismans, "From Harmonization to Coordination? EU law in the Lisbon Governance Architecture", Journal of European Public Policy, 2011, 18 (4), pp. 502-522.

6.Pierre Lascoumes, Patrick Le Galès (dirs.), Gouverner par les instruments, Paris, Presses de Sciences Po, 2004, p. 13.

7. La notion de consensus permissif renvoie à l'acceptation et au soutien implicite de la nature élitiste de l'intégration européenne par les citoyens européens pendant près de 40 ans. L'intégration européenne était, selon cette compréhension citoyenne, issue de la volonté commune des gouvernements des États membres élus démocratiquement et ne pouvait ainsi qu'être démocratique (Leon N. Lindberg, Stuart A. Scheingold, Europe's Would-Be Polity: Patterns of Change in the European Community, Prentice-Hall, Englewood Cliffs), 1970.

8. Karl Raustiala et Anne-Marie Slaughter, International Law, International Relations and Compliance. Princeton Law \& Public Affairs Paper $n^{\circ}$ 02-2, 2012, p. 539.

9. CJCE 5 février 1963 Van Gend en Loos ; CJCE 15 juillet 1964 Costa c/ Enel.

10. Cette demande de conformité est spécifique au niveau international où des États souverains acceptent de participer à une entité supranationale, forme d'intégration dont l'Union européenne est une des illustrations les plus abouties. L'intégration de sociétés abandonnant leur souveraineté dans un État se distingue de cette forme dans la mesure où l'État centralisé ou fédéral possède le monopole de la violence physique légitime. 11. En France, le Conseil d'État a accepté progressivement la jurisprudence de la Cour sur la primauté et l'effet direct et s'est débarrassé des instruments juridiques qu'il avait créés à cet effet. Par exemple, il a abandonné, en 1989, dans l'arrêt Nicolo, la théorie de la loi-écran qui lui permettait de s'opposer à la primauté du droit communautaire sur les lois posté- 
rieures contraires.

12. Gérard Druesne, Droit de l'Union européenne et politiques communautaires, PUF, 2006, coll. Droit fondamental ; Louis Dubouis et Claude Blumann, Droit matériel de l'Union européenne, Domat Montchrestien, 2009 ; Catherine Barnard, The Substantive Law of the EU: The Four Freedoms, Oxford University Press, 2010.

13. Guiseppe Ciavarini Azzi,(ed.), L'application $d u$ droit communautaire par les États membres, European Institute of Public Administration, Maastricht, 1985 ; Samuel Krislov, Claus-Dieter Ehlermann, Joseph Weiler, « The Political Organs and the Decision-Making Process in the United States and the European Community", in Integration Through Law: Europe and the American Federal Experience, Mauro Cappelletti, Monica Seccombe, Joseph Weiler, (eds.), Walter de Gruyter, Berlin, 1986 ; Heinrich Siedentopf, Jacques Ziller, (eds.), Making European Policies Work: The Implementation of Community Legislation in the Member States, Sage, London, 1988.

14. Guiseppe Ciavarini Azzi (ed.), op. cit., 1985, p. 199. 15. En cas de non transposition d'une directive, et comme dans tous les cas où un État n'a pas respecté une des obligations qui lui incombent en vertu des traités, un recours en manquement peut être initié par la Commission européenne sur la base de l'article 258 TFUE. Après avoir mis cet État en mesure de présenter ses observations, la Commission émet un avis motivé auquel l'État doit se conformer. S'il ne le fait pas, la Commission peut saisir la CJUE et lui demander de constater le manquement. Selon l'article 260 TFUE, l'État en question est tenu de prendre, dans les plus brefs délais, les mesures que comporte l'exécution de l'arrêt de la Cour. Dans l'hypothèse où ces mesures ne seraient pas prises, la Commission peut demander à la Cour de condamner l'État récalcitrant au paiement d'une amende ou d'une astreinte.
16. Voir Oliver Treib, "Implementing and complying with EU governance outputs", Living Reviews in European Governance, 2008 : http://www.livingreviews. org/lreg-2006-1 (consulté le 24.8.2012)

17. Gerda Falkner, Oliver Treib, Miriam Hartlapp, Simone Leiber, Complying with Europe: EU Harmonisation and Soft Law in the Member States, Cambridge University Press, Cambridge, 2005 ; Tanja A. Börzel, Tobias Hoffmann and Diana Panke, « Caving in or sitting it out? Longitudinal patterns of non-compliance in the European Union », Journal of European Public Policy, 19(4), 2012, pp. 454-471. Oliver Treib, "Implementing and complying with EU governance outputs", op. cit.

18. Francesco G. Duina, "Explaining Legal Implementation in the European Union", International Journal of the Sociology of Law, 25(2),1997, pp. 155-180 ; Tanja A. Börzel and Thomas Risse, "When Europe Hits Home. Europeanization and Domestic Change, European Integration online Papers (EIoP), 4 (15), 2000, http://eiop.or.at/eiop/texte/2000-015a.htm; Thomas Risse, Maria Green Cowles and James Caporaso (eds), Transforming Europe: Europeanization and Domestic Change Ithaca NY: Cornell University Press, 2001.

19. Adrienne Héritier, Policy-Making and Diversity in Europe, New York, Cambridge University Press, 1999 ; Adrienne Héritier et al., Differential Europe. The European Union Impact on National Policymaking, Lanham, Rowman \& Littlefield, 2001.

20. Pour n'en citer que quelques-uns Gerda Falkner, Miriam Hartlapp, Oliver Treib, "Worlds of Compliance: Why Leading Approaches to European Union Implementation Are Only 'Sometimes-true Theories'”, European Journal of Political Research 3, 2007, pp. 395-416. Gerda Falkner et al., 2005, op. cit. Gerda Falkner, Oliver Treib, "Three Worlds of Compliance or Four? The EU-15 Compared to New Member States", Journal of Common Market Studies, 46(2), 2008, pp. 
293-313; Miriam Hartlapp, Gerda Falkner, "Problems of Operationalization and Data in Eu Compliance Research", European Union Politics, 10(2), 2009, pp. 281-302.

21. Dimiter Toshkov, “Taking Stock: A Review of quantitative studies of transposition and implementation of EU law", EIF Working Paper $n^{\circ} 01.2010$, February 2010.

22. Ellen Mastenbroek, "EU Compliance: Still a Black Hole?", Journal of European Public Policy, 12(6), 2005, pp. 1103-1120; Tanja Börzel, Meike Dudziak, Tobias Hofmann, Diana Panke, Carina Sprungk, "Recalcitrance? Inefficiency, and Support for European Integration: Why Member States do (Not) Comply with European Law", Center for European Studies Working Paper, 2007, $n^{\circ}$ 161, Harvard University, Cambridge; Thomas König and Luetgert Brooke, "Troubles with Transposition? Explaining Trends in Member-State Notification and the Delayed Transposition of EU Directives', British Journal of Political Science 39 (1), 2009, pp. 163-194; Tanja Börzel et al op.cit. 2012.

23. Adapté de Gerda Falkner et al., 2005; op. cit., p. 15. 24. Annette Töller, "Measuring and Comparing the Europeanization of Public Policies", Journal of Common Market Studies, 48(1), 2010, pp. 413-440; Oliver Treib, "Implementing and complying with EU governance outputs", Living Reviews in European Governance, 3 (5), 2008, http://www.livingreviews.org/lreg-2008-5. 25. Gerda Falkner, Miriam Hartlapp, Simone Leiber, Oliver Treib, "Non-Compliance with EU Directives in the Member States: Opposition Through the Backdoor?", West European Politics, 27(3), 2004, pp. 452-473.

26. Esther Versluis, "Even Rules, Uneven Practices: Opening the 'Black Box' of EU Law in Action", West European Politics, 30(1), 2007, pp. 50-67.

27. Adapté de Dimiter Toshkov, 2010, op. cit..
28. Renaud Dehousse (dir), The Community Method. Obstinate or Obsolete? London, Palgrave Macmillan 2012.

29. Liesbet Hooghe et Gary Marks, «A Post-functionalist Theory of EUropean Integration : From Permissive Consensus to Contraining Dissensus », British Journal of Political Science, 39(3), 2008, pp. 1-23.

30. Pour une distinction entre hard law et soft law dans le contexte de l'Union européenne voir Fabien Terpan, "Does EU Soft Law Differ from International Soft Law? Using Soft Law in a Supranational System of Governance, An Agenda for Research", International Studies Association's 53rd Annual Convention, San Diego 1-4 April 2012.

31. Annette Töller, "Voluntary Approaches to Regulation - Patterns, Causes, and Effects", in David LeviFaur (ed), Handbook of the Politics of Regulation, Cheltenham, Edward Elgar, 2011.

32. Bruno Isabelle, Sophie Jacquot and Lou Mandin, "Europeanization through its instrumentation", Journal of European Public Policy, 13(4), 2006, pp. 519-536.

33. Jordan Andrew and Adriaan Schout, Co-ordination of European Governance, Oxford, Oxford University Press, 2006.

34. Renaud Dehousse, "La méthode ouverte de coordination. Quand l'instrument tient lieu de politique", in Pierre Lascoumes and Patrick Le Galès (eds), Gouverner par les instruments, Paris, Presses de Sciences Po, 2004, pp. 331-356 ; Borrás Susana and Thomas Conzelmann, "Democracy, Legitimacy and Soft Modes of Governance in the EU: The Empirical Turn", Journal of European Integration, 29(5), 2007, pp. 531-548. 35. Claudio Radaelli and Anne Meuwese, "Hard Questions and Equally Hard Solutions. Proceduralisation through Impact Assessment in the European Union", West European Politics, 31(1), 2010, pp. 136-153. 36. Annette Töller, "Voluntary Approaches to Regula- 
tion - Patterns, Causes, and Effects", op. cit.

37. Ian Bache and Andrew Taylor, "The Politics of Policy Resistance: Reconstructing Higher Education in Kosovo", Journal of Public Policy, 23(3), 2003, pp. 279-300 .

38. Pierre Lascoumes et Patrick Le Galès, «Définition des instruments de l'action publique », dans Pierre Lascoumes et Patrick Le Galès (eds), Gouverner par les instruments, Paris, Presses de Sciences Po, 2004, 13. 39. Voir Gerda Falkner et al., 2005, op. cit.

40. Jean Pierre Le Bohic et Pierre Lascoumes, « La résistance aux instruments. Formes et modèles d'analyse au sein d'un corpus d'études de cas », Communication $\mathrm{rEr}$ - réseau État recomposé, Les instruments d'action publique, 6 au 8 janvier 2011.

41. En renvoyant à Albert O. Hirschmann, Exit, Voice, and Loyalty: Responses to Decline in Firms, Organizations, and States, Cambridge, MA: Harvard University Press, 1970.

42. George Tsebelis, Nested Games: Rational Choice in Comparative Politics, Berkeley: U.C. Press, 1995.

43. Par ressources sociales nous comprenons les liens que les acteurs administratifs ont pu tisser afin d'accroitre leur pouvoir (liens avec les médias, les décideurs, des réseaux de pouvoir).

44. Nikolaos Zahariadis, "Europeanization as Programme Implementation : Effective and Democratic ?", Journal of Comparative Policy Analysis, 10 (3), 2008, pp. 221-238(18).

\section{Remerciements}

Nous tenons à remercier Virginie Tournay, Amandine Crespy, Charlotte Halpern, Jean Leca et les évaluateurs anonymes pour leurs remarques et suggestions extrêmement pertinentes sur une version antérieure de l'article.

\section{$\mathrm{R} \cdot \dot{E} \cdot \mathrm{S} \cdot \mathrm{U} \cdot \mathrm{M} \cdot \mathrm{E}$}

Depuis une vingtaine d'années, les oppositions des citoyens à l'intégration européenne deviennent plus visibles : manifestations contre des politiques européennes spécifiques, rejet de referendums, abstention accrue aux élections européennes. Une autre forme d'opposition, moins visible, existe depuis le début du projet européen : celle des administrations appelées à mettre en œuvre des décisions européennes, qu'il s'agit de règles de hard law ou de soft law. L'objet de cet article est de revenir de manière critique et dans une perspective historique sur la littérature qui porte sur ces « résistances » actives autant que passives à la mise œuvre des normes européennes. Sur cette base, nous présentons un cadre conceptuel qui permettra d'analyser ces oppositions bureaucratiques de manière systématique. Il s'agit de mieux identifier les types d'instruments utilisés pour « résister » aux normes européennes en prenant comme point de départ l'action de l'administration, considérée comme l'acteur central dans la mise en œuvre des normes européennes.

Abstract
Over the last twenty years, Europeans seem to increas-
ingly oppose European integration: protests against
certain European policies, negative results on refer-
enda, increased abstentions in elections to the European
Parliament. Another form of resistance to European
integration existed however since the 1950s: that of
national administrations who implement European
decisions, whether they are hard or soft law. The aim
of this article is to revisit the forms and instruments
of administrative resistance to implementation in a
historical perspective and to present a conceptual
framework, which will help us to systematically anal-
yse these bureaucratic resistances beyond specific 